\title{
Study Rationale and Design of ADVANCE: Action in Diabetes and Vascular disease - preterax and diamicron MR controlled evaluation
}

\author{
ADVANCE Management Committee* \\ Institute for International Health, University of Sydney, Newtown, Sydney, Australia \\ * see Acknowledgements
}

\section{Abstract}

Aims/hypothesis. Patients with Type II (non-insulindependent) diabetes mellitus are at increased risk of macrovascular and microvascular disease, both of which are reduced by controlling raised blood pressure in hypertensive patients. Intensive glycaemic control has also been shown to reduce microvascular disease but the effects on macrovascular disease remain uncertain. This study will examine the hypotheses that lowering blood pressure with an ACE inhibitor-diuretic combination and intensively controlling gylcaemia with a sulphonylurea-based regimen in high-risk patients with Type II diabetes (both hypertensive and non-hypertensive) reduces the incidence of macrovascular and microvascular disease.

Methods. The study is a $2 \times 2$ factorial randomised controlled trial that will include 10000 adults with Type II diabetes at high risk of vascular disease. Following 6 weeks on open label perindopril-indapamide combination, eligible patients are randomised to continued perindopril-indapamide or matching placebo, and to an intensive gliclazide MR-based glucose control regimen or usual guidelines-based therapy. Primary outcomes are, first, the composite of nonfatal stroke, non-fatal myocardial infarction or cardiovascular death and, second, the composite of new or worsening nephropathy or diabetic eye disease. The scheduled average duration of treatment and follow-up is 4.5 years. The study will be conducted in approximately 200 centres in Australasia, Asia, Europe and North America.

Conclusion/interpretation. ADVANCE is designed to provide reliable evidence on the balance of benefits and risks conferred by blood pressure lowering therapy and intensive glucose control therapy in high-risk diabetic patients, regardless of initial blood pressure or glucose concentrations. [Diabetologia (2001) 44: 1118-1120]

Keywords Diabetes mellitus, blood pressure, blood glucose, cardiovascular disease, diabetic nephropathy, diabetic retinopathy, controlled clinical trial.
Diabetes mellitus is a major, growing health problem worldwide [1]. Patients with Type II (non-insulin-dependent) diabetes mellitus are at substantially increased risks of macrovascular disease including coronary heart disease and stroke [2] and microvascular disease including retinopathy, nephropathy and neur-

Received: 15 March 2001 and in revised form: 18 May 2001

Corresponding author: Prof. Stephen MacMahon, Institute for International Health, University of Sydney PO Box 576, Newtown, Sydney, NSW 2042, Australia, E-mail: s.macmahon@ iih.usyd.edu.au opathy [3]. High blood pressure and poor glycaemic control are common important risk factors for vascular disease in Type II diabetic patients. For both these risk factors, the associations with vascular disease appear continuous, with no defined lower level of blood pressure or haemoglobin $A_{1 \mathrm{c}}$ below which the risks do not continue to decline $[4,5]$.

Randomised trials have shown that lowering the blood pressure of hypertensive patients with diabetes leads to substantial reductions in major vascular events $[6,7]$ and there seem to be greater benefits of more intensive blood pressure lowering. [8] In addition, the Heart Outcomes Prevention Evaluation 


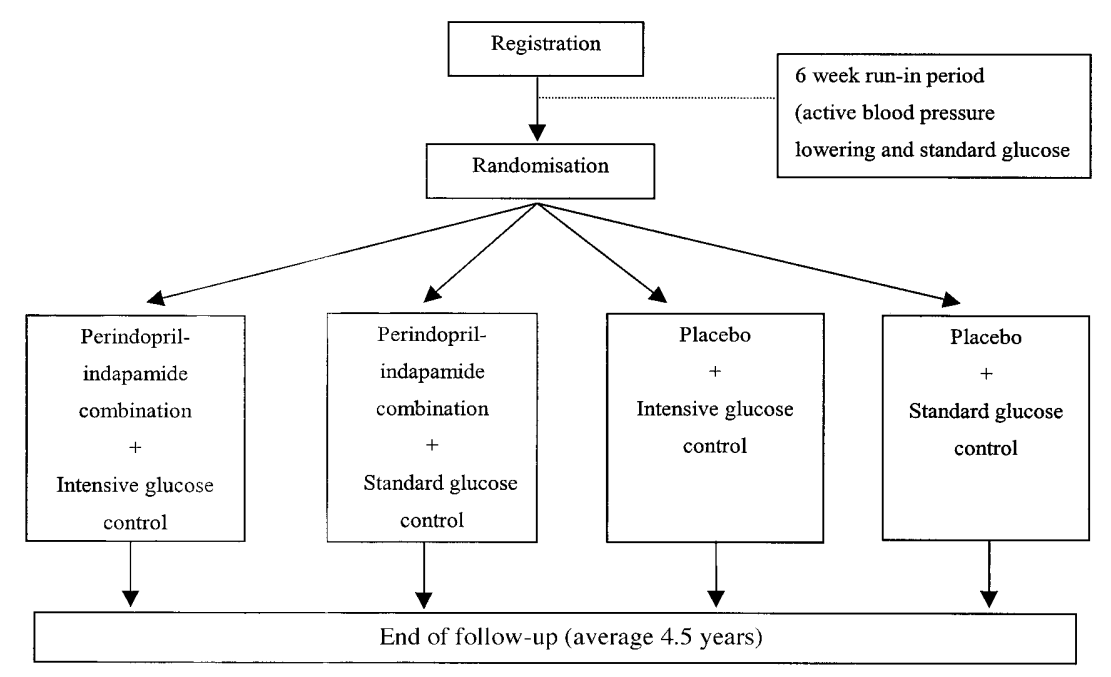

Fig. 1. Study schema

(HOPE) study has shown that ACE inhibitor therapy could confer worthwhile vascular benefits among diabetic patients whether hypertensive or not, even when the reduction in blood pressure is modest [9]. Fewer studies have investigated the effects of intensive glycaemic control on vascular disease risk. In the United Kingdom Prospective Diabetes Study (UKPDS), such treatment was shown to reduce the risk of major microvascular outcomes among patients with newly-diagnosed Type II diabetes, but the effects on macrovascular endpoints were less obvious [10].

Hence, several issues regarding the effects of treatments for blood pressure lowering and glucose control on the risks of vascular disease among patients with Type II diabetes remain unresolved. First, are there worthwhile benefits of blood pressure lowering when provided routinely to high-risk diabetic patients regardless of the blood pressure? Second, are any such benefits additional to those conferred by background treatment with an ACE inhibitor? Third, does intensive glucose control therapy targeting low concentrations of haemoglobin $\mathrm{A}_{1 \mathrm{c}}$ reduce the risk of major macrovascular disease and confer greater protection against microvascular disease? ADVANCE (Action in Diabetes and Vascular Disease: Preterax and Diamicron MR Controlled Evaluation) is a large-scale, $2 \times 2$ factorial, randomised controlled trial that has been designed specifically to address each of these issues.

\section{Subjects and methods}

Subjects. The study will include at least 10000 adults with Type II diabetes recruited from approximately 200 clinical centres in Australasia, Asia, Europe and North America. Patient entry criteria have been designed to facilitate the enrolment of a broad cross-section of patients at high risk. Eligibility for the trial is not-dependent on blood pressure or the use of other blood-pressure lowering therapy, nor is it dependent upon the entry concentration of haemoglobin $\mathrm{A}_{1 \mathrm{c}}$ or fasting blood glucose or the type or number of oral agents used for glucose control. Additionally, eligibility is not dependent upon the need for or use of ACE-inhibitor therapy.

Patients are eligible for the study if they had their first diagnosis of Type II diabetes mellitus at age 30 years or older, are age 55 or older at entry, and are at a substantially high risk of vascular events. The latter is indicated by a diagnosis of Type II diabetes made 10 or more years before entry, or age 65 years or older at entry, or a history of any of the following: stroke or myocardial infarction, hospital admission for transient ischaemic attack or unstable angina, coronary or peripheral revascularisation, amputation secondary to vascular disease, macroalbuminuria, proliferative retinopathy or retinal photocoagulation therapy, macular oedema, blindness in one eye thought to be caused by diabetes, or another major risk factor for vascular disease (defined as current cigarette smoking, total cholesterol $>6.0 \mathrm{mmol} / \mathrm{l}$, HDL cholesterol $<1.0 \mathrm{mmol} / 1$ or microalbuminuria). $\mathrm{Pa}$ tients with a definite indication for long-term insulin therapy are not eligible for inclusion. Patients for whom an ACE inhibitor is indicated are eligible, unless there is a specific indication for an ACE inhibitor other than perindopril at a dose of 2 or $4 \mathrm{mg}$ daily.

Study treatment. The blood pressure lowering treatment chosen is a fixed low-dose combination of perindopril $(2-4 \mathrm{mg})$ and indapamide $(0.625-1.25 \mathrm{mg})$. This combination of an ACE inhibitor and diuretic was selected because of the established effects of both classes of drug on cardiovascular disease risks in various patient populations, and the greater blood pressure lowering effects of combination therapy compared with monotherapy. For any patient in whom an ACE inhibitor is thought to be indicated, background perindopril (open-label; 2 or $4 \mathrm{mg}$ daily) will be provided and can be started at any time during the study. Whenever required, other classes of blood pressure lowering drugs could be prescribed at the discretion of the responsible clinician.

The glucose control regimen chosen is based on a modifiedrelease sulphonylurea preparation (gliclazide MR 30-120 mg). Sulphonylureas are widely prescribed for blood glucose control in diabetes and the modified release gliclazide formulation provides 24-h glucose control in a single daily dose. Non-pharmacological therapy, other oral agents and then insulin can be 
added as required to achieve the target concentration of haemoglobin $A_{1 c}$ (6.5\% or less).

After a 6-week run-in period on open fixed low-dose perindopril-indapamide $(2.0 \mathrm{mg} / 0.625 \mathrm{mg})$ and usual glucose lowering treatment, eligible participants will be randomly assigned in a factorial design to the two treatment comparisons (Fig. 1): a double blind comparison of the perindopril-indapamide combination (initially $2.0 \mathrm{mg} / 0.625 \mathrm{mg}$ increasing to $4.0 \mathrm{mg} /$ $1.25 \mathrm{mg}$ daily after 3 months) compared with matching placebo; and an open comparison of gliclazide MR-based intensive therapy (target haemoglobin $\mathrm{A}_{1 \mathrm{c}} \leq 6.5 \%$ ) compared with therapy for glucose control based on standard guidelines.

Randomisation. A central, computer-based randomisation service will assign patients to treatments stratified by the study centre, history of serious macrovascular disease or microvascular disease and background use of perindopril at baseline.

Outcomes. There are two primary outcomes: first, the composite of non-fatal stroke, non-fatal myocardial infarction or cardiovascular death; and second, the composite of new or worsening nephropathy or microvascular eye disease. An Endpoint Adjudication Committee blind to treatment assignment, will review all suspected primary outcomes. Secondary outcomes include cerebrovascular disease, coronary heart disease, heart failure, peripheral vascular disease, microalbuminuria, visual deterioration, neuropathy, dementia, and all-cause mortality. Data will also be collected on episodes of major and minor hypoglycaemia, other suspected serious adverse reactions, as well as quality of life and use of health care.

Follow-up. The scheduled average post-randomisation duration of follow-up will be 4.5 years. Those assigned the gliclazide MR-based intensive glucose lowering regimen will be seen at least once every 3 months, while those randomised to standard guidelines-based therapy will be seen once every 6 months for most of the scheduled follow-up period.

Statistical considerations. The study has been designed to provide at least $90 \%$ power (with $\alpha=0.05$ ) to detect a $16 \%$ or greater reduction in the relative risk of each of the primary outcomes in each of the randomised comparisons. This assumes a $3 \%$, or more, annual event rate for each of the two primary outcomes among participants assigned the control condition.

The main effects and interactions of the study treatments for each of the primary outcomes will be determined using Cox models. Analyses of secondary outcomes will be conducted using standard statistical procedures applicable to dichotomous, categorical or continuous data as appropriate. All study outcomes will be analysed on the basis of intention to treat. An independent Data and Safety Monitoring Committee will review unblinded safety and outcome data at regular intervals during follow-up.

\section{Study organisation and timeline}

International coordination will be provided by the Institute for International Health in Sydney, Australia with central data management provided by the Clinical Trials Research Unit in Auckland, New Zealand. Regional coordination will be provided by five major regional coordinating centres (in Beijing, London, Melbourne, Montreal and Utrecht). Recruitment commenced in June 2001 and is expected to be com- pleted in September 2002. Follow-up will continue until June 2006, with publication of final results anticipated early in 2007.

Acknowledgements. ADVANCE is an investigator-initiated and -conducted study, funded by a grant from the Institut de Recherches Internationales Servier. Members of the Management Committee: J. Chalmers*, Chairman, Australia; M. Cooper, Australia; E. Ferrannini*, Italy; P. Glasziou, Australia; D. Grobbee, Netherlands; P. Hamet, Canada; S. Harrap, Australia; L. Liu, People's Republic of China; S. MacMahon*, Vice-Chairman, Australia; G. Mancia, Italy; M. Marre, France; D. Matthews, United Kingdom; C. Mogensen, Denmark; B. Neal*, Australia; C. Yu Pan, People's Republic of China; N. Poulter, United Kingdom; A. Rodgers, New Zealand; B. Williams, United Kingdom; M. Woodward, Australia. Members of the Data and Safety Monitoring Committee (United Kingdom): R. Collins, P. Sandercock, P. Sleight, R. Holman. Members of the Endpoint Adjudication Committee (Australia): G. Fulcher, C. Pollock, D. Celermajer, J. Watson, B. Harrisberg. Staff of the International Coordinating Centre (Australia): R. Currie, S. Girgis*, K. Jayne, H. Monaghan, A. Patel*, A. Richens. Staff of the Data Management Centre (New Zealand): B. Gray, A. Milne. Staff of the Regional Coordinating Centres: A. Adderkin, United Kingdom; A. Bak, Netherlands; S. Flett, Australia; D. de Guise, Canada; E. Jetses, Netherlands; J. Reid, Australia; R. Stolk, Netherlands; W. Wang, China; F. Williams, Australia; X. Zou, China. *Members of the Writing Subcommittee.

\section{References}

1. King H, Aubert R, Herman W (1998) Global burden of diabetes, 1995-2025: Prevalence, numerical estimates, and projections. Diabetes Care 21: 1414-1431

2. Stamler J, Vaccaro O, Neaton JD, Wentworth D (1993). Diabetes, other risk factors, and 12-yr cardiovascular mortality for men screened in the Multiple Risk Factor Intervention Trial. Diabetes Care 16: 434-444

3. Nathan DM (1993) Long-term complications of diabetes mellitus. N Engl J Med 328: 1676-1685

4. Adler A, Stratton IM, Neil H et al. (2000) Association of systolic blood pressure with macrovascular and microvascular complications of type 2 diabetes (UKPDS 36) prospective observational study. BMJ 321: 412-419

5. Stratton I, Adler A, Neil H et al. (2000) Association of glycaemia with macrovascular and microvascular complications of type 2 diabetes (UKPDS 35): prospective observational study. BMJ 321: 405-412

6. Lièvre M, Gueyffier F, Ekbom T et al. (1997) Efficacy of diuretics and beta-blockers in diabetic hypertensive patients: results from a meta-analysis. Diabetes Care 20: 621-622

7. UK Prospective Diabetes Study Group (1998) Tight blood pressure control and risk of macrovascular and microvascular complications in type 2 diabetes: UKPDS 38. BMJ 317: 703-713

8. Blood Pressure Lowering Treatment Trialists' Collaboration (2000) Effects of ACE inhibitors, calcium antagonists, and other blood-pressure-lowering drugs: results of prospectively designed overviews of randomised trials. Lancet 355: 1955-1964

9. Heart Outcomes Prevention Evaluation (HOPE) Study Investigators (2000) Effects of ramipril on cardiovascular and microvascular outcomes in people with diabetes mellitus: results of the HOPE study and MICRO-HOPE substudy. Lancet 355:253-258

10. UK Prospective Diabetes Study (UKPDS) Group (1998) Intensive blood-glucose control with sulphonylureas or insulin compared with conventional treatment and risk of complications in patients with type 2 diabetes (UKPDS 33). Lancet 352: 837-853 